University of Nebraska - Lincoln

DigitalCommons@University of Nebraska - Lincoln

Faculty Publications in Computer \& Electronics Electrical \& Computer Engineering, Department Engineering (to 2015)

2008

\title{
Non-Optimal receiver for Space Time Spreading across a Time Hopping over Ultra Wideband PPM
}

\author{
Peter Vial \\ University of Wollongong \\ Beata Wysocki \\ University of Nebraska-Lincoln \\ Tadeusz Wysocki \\ University of Nebraska-Lincoln, wysocki@uow.edu.au
}

Follow this and additional works at: https://digitalcommons.unl.edu/computerelectronicfacpub

Part of the Computer Engineering Commons

Vial, Peter; Wysocki, Beata; and Wysocki, Tadeusz, "Non-Optimal receiver for Space Time Spreading across a Time Hopping over Ultra Wideband PPM" (2008). Faculty Publications in Computer \& Electronics Engineering (to 2015). 16.

https://digitalcommons.unl.edu/computerelectronicfacpub/16

This Article is brought to you for free and open access by the Electrical \& Computer Engineering, Department of at DigitalCommons@University of Nebraska - Lincoln. It has been accepted for inclusion in Faculty Publications in Computer \& Electronics Engineering (to 2015) by an authorized administrator of DigitalCommons@University of Nebraska - Lincoln. 


\section{Non-Optimal receiver for Space Time Spreading across a Time Hopping over Ultra Wideband PPM}

Peter Vial, Beata Wysocki \& Tadeusz A Wysocki

University of Wollongong

School of Electrical, Computer and Telecommunications Engineering

Email: \{Peter_Vial,Beata_Wysocki,Tad_Wysocki\}@uow.edu.au

\begin{abstract}
A novel non-optimal chip based technique is proposed to use two antennas to transmit data across a Direct Sequence Time Hopping Ultra Wideband dual antenna wireless communication system to a single receiver. The spread data is sent over the independent channels using the Space Time Spreading technique to encode the chips which are sent on each antenna simultaneously. A hard decision is made at each chip of the spreading code received based on the known Channel State Information which Pulse Position Modulated signal was received from each antenna. The paper shows that by adapting the Space Time Spreading technique to use three pulse positions in a dual input single output system one can transmit data at a lower Bit Error Rate for high $\mathrm{Eb} / \mathrm{No}$. Three receive techniques are considered; the use of the first arriving rays, the first set of useful arriving rays and the best set of arriving rays for each symbol period. The proposed receiver is less complex than that of the optimal system where a Maximum Ratio Combiner is used across the entire spread signal space (per symbol rather than per chip), requiring more memory storage in the receiver. In addition, symbols are transmitted during the period of one symbol period for a single antenna system. This is achieved at the expense of a higher measured BER compared to single antenna systems.
\end{abstract}

Keywords: Space Time Spreading, MIMO, SISO, SalehValenzuela, BER, UWB, MRC, SRake, PRake, Direct Sequence, Time Hopping, Walsh-Hadamard

\section{INTRODUCTION}

In our previous work [1][2], we have covered the development of Space Time Spreading (STS) systems in the presence of Multiple Access Interference (MAI) and the development of a Direct Sequence Time Hopping Ultra Wideband Pulse Position Modulation (DS-TH-UWB-PPM) simulation using MATLAB's SIMULINK. Here we combine these two systems (STS and DS-TH-UWB-PPM) across a Multiple Input Multiple Output (MIMO) wireless link and combine its performance with the equivalent Single Input Single Output (SISO) DS-TH-UWB-PPM system. In both schemes we implement the MRC (Maximum Ratio Combiner) detector on a chip by chip basis rather than a symbol by symbol basis. This system is simpler to implement, at the loss of overall channel information in a MIMO system that uses a symbol by symbol decoder. By using the proposed technique, at high $\mathrm{E}_{\mathrm{b}} / \mathrm{N}_{\mathrm{o}}$, the data rate can be effectively doubled compared to a SISO based equivalent system using only DS-TH-UWB-PPM [3][2]. This is achieved, however, at the cost of an increase in observed BER.

This paper is organised as follows. In Section II we outline the simulation of the DS-TH-UWB two pulse position simulation (as already described in [3]). In Section III we propose how the two pulse position simulation can be modified to three pulse positions using the STS Technique proposed in [4] as applied across two SV (Saleh-Valenzuela) channels (one for each transmitting antenna) using UWB PPM. In Section IV we outline how the Symbol Energy to Noise $\left(E_{b} / N_{o}\right)$ ratio was measured in the SIMULINK simulation. In Section $\mathrm{V}$ we present the simulation results and compare the results for the same seed value between the SISO and MIMO system proposed here, while Section VI concludes the paper and presents areas for future research.

\section{DS-TH-UWB WITH TWO PULSE POSITIONS}

The Simulation of a SISO DS-TH-UWB-PPM system as described in [3] and [2] was re-used for comparison with the STS dual antenna MIMO system proposed in this paper. The system described in [3] and [2] used a Rake based receiver to collect the energy of each finger followed by a chip based MRC detector. These simulation results are reproduced from [3] using the same seed value for the comparison of the two systems. The system described in Section III uses a dual antenna single receiver system (MIMO) whereas the results found in [3] used single antenna to single antenna or SISO based system. The system described in [3] used an outer loop spreading / de-spreading stage on the chip by chip waveforms that were received for the two pulse position systems. The same time hopping pattern was used in all cases which was randomly chosen initially but is fixed at $\left[\begin{array}{llllllll}6 & 0 & 5 & 3 & 6 & 4 & 5 & 3\end{array}\right]$ so each system can be appropriately compared. The spreading / de-spreading sections used a Walsh-Hadamard thirty two chip code. The time hopping pattern used was split into 8 slots, numbered 0-7. Each slot was fixed at one hundred nano-seconds. Only one of the eight slots is used to transmit the chip. For example, in the first eight slots, slot number six was used to transmit the two pulse position UWB signal [3].

The SISO system had its multipath channel generated using MATLAB files with different seed values based on the SV real channel coefficients described in [5] and [6]. These MATLAB files pre-ranked the Gaussian multipath rays received in terms of relative time displacement between rays and relative strength of the rays. Within the simulation for the same seed value the algorithms used were to look at the first $\mathrm{L}$ arriving rays, the first $\mathrm{L}$ arriving rays which are one nanosecond away from other multipath, and the strongest L arriving rays which are at least one nanosecond away from 
other multipath rays. We did this in [3] for $L=1$ and $L=4$. The results reported in [3] are re-produced in Figure 1a.

\section{MIMO SYSTEM}

In [3] it was observed for the STS based modified DS-THUWB-PPM simulation that in simulations of the STS baseband systems described in [7] one could represent at the chip level values being sent by $-2,0,2$. It was suggested in [3] that these could be represented by three time shifts of the Gaussian pulses used for transmission. This added only the addition on one extra time shift. Thus by adding only one extra pulse position it was feasible to send two symbols in the same period that only a single symbol was sent using DS-THUWB-two position PPM. This was achieved with perfect knowledge of the channel characterization and timing at the receiver in the presence of noise on a chip by chip basis [3]. Further, it was observed in the simulation of the STS baseband system [7] that each channel had a different discrete value transmitted. That is, if a -2 was sent on one antenna path a 0 or 2 (but not a -2) was transmitted on the other. For a system based on pulse position modulation, where Gaussian pulses are transmitted at different time shifts to represent $-2,0$, and 2 , each antenna would transmit a pulse at a slightly different time. If we use orthogonal pulse position modulation and the UWB channel characteristics of each transmitting antenna are uncorrelated, it will lead to the receiver (having perfect Channel State Information (CSI)) being able to detect and recover the data stream originally transmitted.

With the STS technique applied to DS-TH-UWB PPM we transmit the sum stream on antenna 1 and the difference stream on antenna 2. Since its now over two antennas we refer to it as STS-TH-UWB-PPM. Each antenna is assumed to correspond to an uncorrelated SV channel (using two different seed values as used in [3]). This can provide a diversity of up to two at the transmitters (space diversity). These streams involve the use of two 32 bit spreading sequences. As in [3] we represent the individual chips of these spreading sequence by $c_{1}[j]$ and $c_{2}[j]$ where these are the two Walsh-Hadamard spreading sequences used in STS and $\mathrm{j}$ is the counter for the individual chips within that sequence which here is an integer in the range $\{1,2, \ldots, 32\}$. We proceed as in [3] to say that $b_{1}$ and $\mathrm{b}_{2}$ are the two bits to be transmitted across the STS-TH-UWBPPM MIMO channel. Hence for the addition stream we have [3]:

$$
b_{1} c_{1}[j]+b_{2} c_{2}[j]=\left\{\begin{array}{c}
2 \\
0 \\
-2
\end{array}\right\} \quad \text { for } \quad j=1, \ldots, 32
$$

while for the difference stream we have [3]:

$$
b_{2} c_{1}[j]-b_{1} c_{2}[j]=\left\{\begin{array}{c}
2 \\
0 \\
-2
\end{array}\right\} \quad \text { for } \quad j=1, \ldots, 32
$$

The stream of Equation 1 is then transmitted on antenna one. The stream of Equation 2 is transmitted on antenna two. To normalize the system for a two antenna transmission, each antenna transmits at half the power of a SISO system (that is, we multiply the signal amplitudes by $1 / \sqrt{2}$ in the simulations). Either of these streams can then be transmitted and demodulated at the single receiver in the presence of Gaussian noise using the Space Time Spreader demodulation technique used in [7]. This means that one symbol now represents two bits instead of the one bit represented per symbol transmitted in a system described in Section II. This is achieved at the cost of an extra PPM time shift. Table 1 shows the STS codes for individual chip level values for 32 chip spreading sequences $\mathrm{c}_{1}$ and $c_{2}$ used in the SIMULINK/MATLAB simulation. This technique can also be applied to MIMO systems with four and eight transmit antennas and a single receive antenna. To apply it to such scenarios requires five pulse position shifts for four antennas and nine pulse positions for eight transmit antennas. These are the only situations $\left(\mathrm{n}_{\mathrm{T}}=2,4\right.$ and 8$)$ where STS full rate systems can be used [8]. For these cases we require $n_{T}+1$ pulse positions. The proof that these are also valid can be shown in the same way as the formation of Table 1 . That is, form the chip values sent for each antenna in use and tabulate against possible values for the $b_{i}$, where $i$ ranges from 1 to 4 for $\mathrm{n}_{\mathrm{T}}=4$ and 1 to 8 for $\mathrm{n}_{\mathrm{T}}=8$.

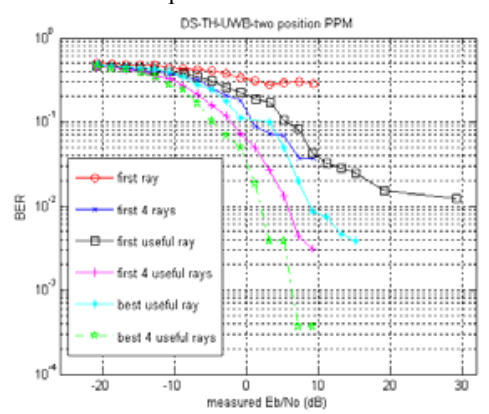

(a)

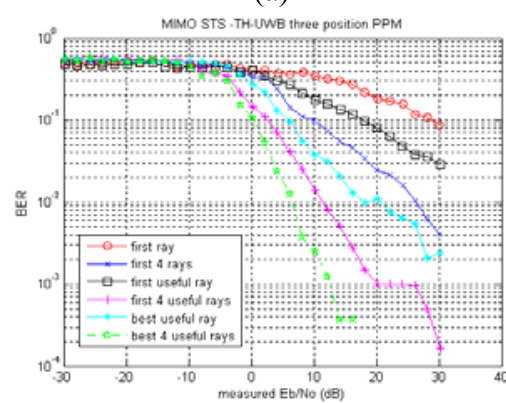

(b)

Figure 1: (a) DS-TH-UWB simulations for 2 position PPM [3]

(b) STS Performance of MIMO TH-UWB- 3 position PPM proposed chip based MRC (non-optimal)

Using the same UWB channels as described in Section II we conducted the simulations for the first ray, first useful ray ( $\mathrm{L}=1$, Partial Rake), the best useful ray ( $\mathrm{L}=1$, Selective Rake), the first four rays, the first four useful rays ( $\mathrm{L}=4$, Partial Rake) and the best four useful rays $(\mathrm{L}=4$, Selective Rake). The obtained results are shown in Figure 1a [3]. We also performed this simulation for $\mathrm{n}_{\mathrm{T}}=2$ and a single receiver case but the channels that were used were two uncorrelated SV channels for each transmit antenna. We did this for the same 
seed values utilized in SISO for one of the antenna channels used; the other channel had a different seed value, of course uncorrelated. The results for this are shown in Figure $1 \mathrm{~b}$.

\section{MEASURED E $E_{\mathrm{B}} / \mathrm{N}_{\mathrm{O}}$ FOR MIMO SYSTEM}

The simulator for the MIMO based system incorporated a factor of 0.707 on each antenna as explained in Section III. The mean or average of each antennas total power versus measured noise energy taken over the total slot was then measured (across all multipath in the channel). The result was multiplied by 32 (the number of chips in a symbol) and represented the total energy that was used to transmit two symbols, on average, over one antenna. This was then adjusted for each antenna, considering with STS that each channel transmits both symbols at the same time, one being the sum the other the difference in the case of two transmit antennas. The resulting average measured $\mathrm{E}_{\mathrm{b}} / \mathrm{N}_{\mathrm{o}}$ for the MIMO system was used in the performance results. No such adjustment is done for the SISO system as it transmits only one symbol at a time. A dB Gain SIMULINK module was used in the path of both antennas to change the measured $\mathrm{Eb} / \mathrm{No}(\mathrm{dB})$ in two $\mathrm{dB}$ increments for this system. Table 2 shows sample calculations. All calculations were performed in excel spreadsheets.

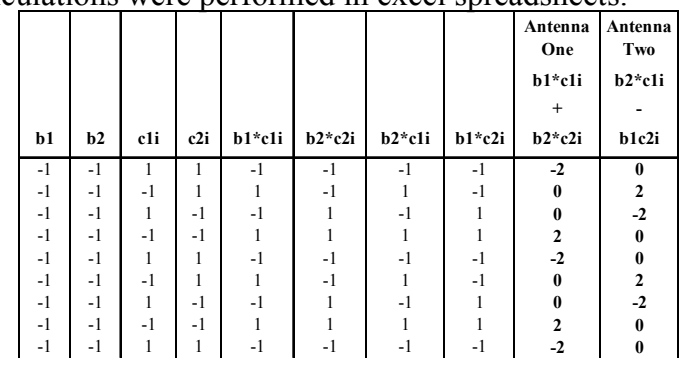

Table 1: Sample of Space Time Spreading codes shown for individual chip level values for a 32 chip spreading sequences $\mathrm{c}_{1}$ and $\mathrm{c}_{2}$ used in simulation when transmitting $\{-1,-1\}$ pairing.

\section{COMPARISON OF MIMO AND SISO}

We now compare the results obtained using the two pulse position UWB described in [3] and Section II and the MIMO STS technique described in Section III. Figure 2a shows the SISO technique outlined in [3] and Section II for the first useful ray and the MIMO system using two antennas outlined in Section III. They show BER versus measured Eb/No (dB). Clearly the system described in Section II provides a lower BER for the same measured Eb/No by about $6 \mathrm{~dB}-20 \mathrm{~dB}$ for high measured $\mathrm{Eb} / \mathrm{No}$. This should be considered in that the MIMO technique transmits two symbols in the same period that the SISO technique transmits a single symbol. Both techniques used a chip by chip basis for the MRC detector with $\mathrm{L}=1$ finger for the Rake receiver and the de-spreading was performed based on the Walsh Hadamard spreading codes employed. Two orthogonal codes are required for the MIMO system and only one for the SISO system.

\begin{tabular}{|c|c|c|}
\hline $\begin{array}{l}\text { SNR } \\
\text { (dB) }\end{array}$ & 2Eb/No (dB) & measured $\mathrm{Eb} / \mathrm{No}(\mathrm{dB})$ \\
\hline 0 & -23.76 & -29.785 \\
\hline 2 & -21.77 & -27.79 \\
\hline
\end{tabular}

system

Figure $2 \mathrm{~b}$ shows the same data as that for Figure $2 \mathrm{a}$ except now the number of fingers used in the Rake receiver is $\mathrm{L}=4$, for the same seed value. For this situation the STS $\mathrm{L}=4 \mathrm{MIMO}$ system has a displacement of approximately 3-6 dB for high measured $\mathrm{Eb} / \mathrm{No}(\mathrm{dB})$. The curves are closer than in the case for $\mathrm{L}=1$ but the SISO system still has a better performance. Figure $2 \mathrm{c}$ shows the results when the best useful rays (or selective rake) are used to detect the symbol sent on a chip by chip basis. Once again the SISO system is $2-5 \mathrm{~dB}$ 's better than the STS based system. Figure $2 d$ shows the results when the best four $(\mathrm{L}=4)$ useful rays are used in the Rake receiver on a chip by chip basis, showing similar performance as in the $\mathrm{L}=1$ situation. From the results the poorer performance of the dual antenna system compared to single antenna system can be attributed to the fact that the chip by chip basis of the decision removes the advantage of knowing the channels exact coefficients and timing over all the energy of the symbol (comprised of 32 Gaussian pulses or chips). This has very little effect on the SISO system as there is only one transmit antenna, however in the MIMO system with two transmit antennas, both uncorrelated channels can interfere with each other. By making a hard decision on a chip by chip basis instead of a symbol by symbol basis, the energy employed in the decision is in fact much smaller than would be used over the whole symbol.

Having two channels (one from each antenna) tends to cause an increase in the probability that a Gaussian pulse on one antenna will coincide with a pulse on the other antenna (as the rays in use will be uncorrelated between the two antennas). Simulations from other studies have shown that much better performance is achievable when all the energy of a symbol is accumulated and used in the hard decision resulting in a symbol being decided upon rather than the chip by chip basis considered here. The major advantage of making a chip by chip hard decision is the fact that the receiver is simpler to implement. It should also be noted that the STS dual antenna system proposed here still enjoys the advantage that two symbols or binary bits are sent in the same period that only one symbol or binary bit is transmitted by the SISO system. Also, if a fade occurs on one antenna it is unlikely to occur on the other and both binary bits are available on either antenna using the STS technique. 


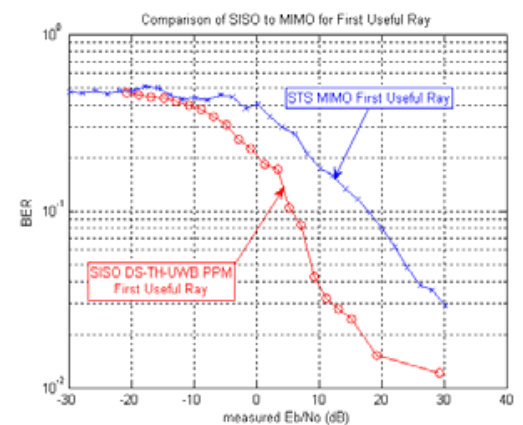

(a)

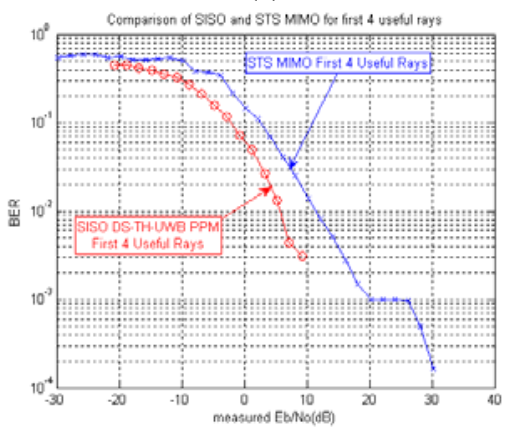

(b)

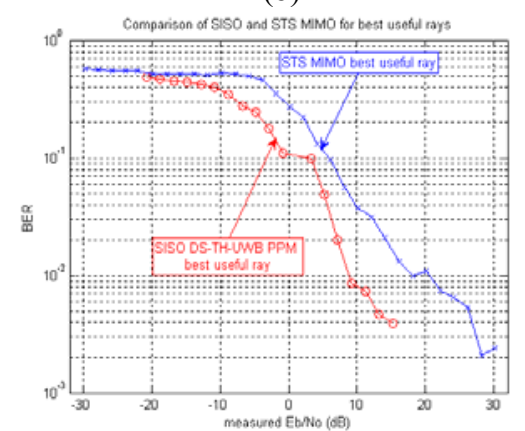

(c)

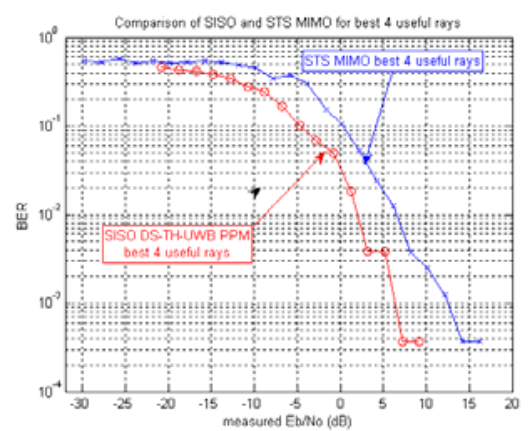

(d)

Figure 2: Comparison of the SISO and MIMO technique for:(a) \& (b) PRake, L=1,4 and (c) \& (d) SRake, L=1, 4

\section{CONCLUSIONS}

This paper illustrates the use of a novel non-optimal technique employing two antennas in a TH UWB PPM MIMO scheme adapted for UWB using an STS technique. This is compared to a SISO based system described in [3]. Both systems make their decisions on a chip by chip basis within a spreading code or codes. It was found that the SISO system had a better performance compared to the MIMO STS based system by between $2 \mathrm{~dB}-20 \mathrm{~dB}$ depending on the number of fingers used in the Rake receiver and the partial or selective rake techniques employed. This needed to be considered in the light that the MIMO STS based system transmits two symbols in the same time that the SISO system can transmit only one such symbol. In fact, it was pointed out that the MIMO technique employed here can be expanded to systems with four and eight transmit antennas where four and eight symbols respectively could be transmitted in the same time that the SISO transmits one symbol. It was suggested that the MIMO system suffers from extra collisions between each antennas uncorrelated channel coefficients and lower transmit power, as shown in the discussion on how $\mathrm{E}_{\mathrm{b}} / \mathrm{N}_{\mathrm{o}}$ is measured (Section IV). This adversely affects the MIMO system compared to the SISO system which has only one multipath channel to transmit the symbols through. It is suggested that improvement would be obtained by employing the MRC detector across an entire symbol rather than over a chip period. However, the presented here non-optimal technique can be used for high measured $\mathrm{Eb} /$ No to provide a transmission technique to send symbols at BER that decreases as measured $\mathrm{Eb} / \mathrm{No}$ is increased. This is simpler to implement compared to the system which uses an $\mathrm{MRC}$ detector across an entire symbol. The simplicity of the demodulator comes at the expense of poorer performance.

\section{REFERENCES}

[1] Peter James Vial, Ibrahim Raad, Tad Wysocki, "On the effect of adjacent sector Multiple Access Interference on Space Time Spreading Systems", DSPCS'03, Coolangatta Queensland, 8-11th of December, 2003.

[2] Peter Vial, Beata Wysocki, Tad Wysocki, "An Ultra Wideband Simulator Using MATLAb/Simulink", DSPCS'05 \& WITSP'05, Noosa Heads, Queensland, 18-21st of December, 2005.

[3] Vial, P. J., Wysocki, T. A. \& Wysocki, B., "Direct Sequence Modified Time Hopping PPM over Ultra Wideband S-V Channel", The 5th Workshop on the Internet, telecommunications and Signal Processing (WITSP'06), Hobart, Tasmania, Australia, 11-14th December 2006

[4] Choi, J.D and Stark, W.E., "Performance analysis of RAKE receivers for ultra-wideband communications with PPM and OOK in multipath channels", IEEE Conference on Communications, vol.3, pp. 1969 1973, 28 April-2 May 2002.

[5] Bertrand Hochwald, Thomas L Marzetta, Constantinos B Papadias, "A Transmitter Diversity Scheme for Wideband CDMA Systems Based on Space-Time Spreading," IEEE Journal on selected areas in communications, Vol 19, No 1, pp. 48-60, January 2001.

[6] A. A. M. Saleh and R. A. Valenzuela, "A statistical model for indoor multipath propagation", IEEE Journal on Selected Areas in Communications, Vol. 5, No. 2, pp. 128-137, Feb. 1987.

[7] L. Yang, and G. B. Giannakis, "Analog Space-Time Coding for MultiAntenna Ultra-Wideband Trasmission", IEEE Transactions on Communications, Volume 52, Issue 3, pp.507-517, March 2004, Digital Object Identifier 0.1109/TCOMM.2004.823644

[8] Peter Vial, Ibrahim Raad, Tad Wysocki, "On Design of a STS channel using Simulink", Radio Elektronica, Bratislava, Slovak Republic, 27-28 April, 2004. 